\title{
El conocimiento grupal de agentes epistémicamente responsables
}

Group Knowledge of Epistemically Responsible Agents

*Eleonora Cresto

\begin{abstract}
Resumen: Este trabajo examina el concepto de conocimiento grupal. Por un lado, se observa que a menudo nos vemos compelidos a atribuir a los agentes grupales actitudes epistémicas que difieren de las de sus miembros individuales; por otro lado, también notamos que los agentes idealmente responsables están sometidos a una "presión deflacionaria" para anclar tales actitudes en individuos concretos. En vistas de ello, argumento que los grupos epistémicamente responsables deben concebirse como entidades dinámicas; de manera correlativa, el conocimiento grupal puede definirse en términos de una familia de posibles rutas entre el conocimiento distribuido y el conocimiento común del grupo. Muestro entonces que las Lógicas de Anuncios Públicos pueden usarse en este contexto como herramientas adecuadas para monitorear la satisfacción de requerimientos normativos por parte de individuos epistémicamente responsables.
\end{abstract}

Palabras-clave: Conocimiento común. Conocimiento distribuido. Lógicas de anuncios públicos. Responsabilidad epistémica.

\begin{abstract}
This paper examines the concept of group knowledge. I start by noticing that we often seem compelled to attribute epistemic attitudes to groups, even when those attitudes differ from the ones held by their individual members. However, it is also the case that ideally responsible agents are subjected to a 'deflationary pressure' to ground those attitudes in concrete individuals. In the light of this, I argue that epistemically responsible groups should be conceived of as dynamic entities. Correlatively, group knowledge can be defined in terms of a family of possible paths between distributed and common knowledge. Finally I show that the Logic of Public Announcements can be used in this context as adequate tool to monitor the satisfaction of normative requirements by epistemically responsible individuals.
\end{abstract}

Keywords: Common Knowledge. Distributed Knowledge. Logic of Public Announcements. Epistemic Responsibility.

\footnotetext{
* Vidt 1986, 1A. Buenos Aires 1425, Argentina. CONICET, Universidad de Buenos Aires/UNTREF. <eleonora.cresto@gmail.com>.
} 
Hn este trabajo presentaré algunas reflexiones sobre el concepto de conocimiento y responsabilidad grupales, así como sobre la naturaleza de la agencia grupal. La propuesta es una continuación de un proyecto anterior sobre la idea de transparencia epistémica, responsabilidad y conocimiento individual. Comenzaré pues por recordar brevemente en qué consiste dicho proyecto, y luego me enfocaré en el conocimiento grupal. Argumentaré que el desideratum de responsabilidad epistémica grupal inmediatamente impone nuevos desiderata sobre los individuos idealmente responsables; en última instancia, argumentaré que ello nos revela la naturaleza esencialmente inestable (o dinámica) de los agentes grupales. Esta concepción sobre los grupos, a su vez, será el hilo conductor para llegar a una definición satisfactoria de conocimiento grupal. Propondré aquí además que las "Lógicas de Anuncios Públicos" (Public Announcement Logics) pueden funcionar como herramientas apropiadas para monitorear la satisfacción de requerimientos normativos por parte de individuos responsables.

En la Sección 1 propondré algunas consideraciones sobre conocimiento y responsabilidad individuales. En la Sección 2 presentaré un marco formal para razonar sobre agentes grupales. Por razones de simplicidad expositiva usaré como base el modelo bien conocido de Fagin et alii (1995) ${ }^{1}$; en diversas notas al pie indicaré posibles maneras de 'traducir' algunas afirmaciones a un formalismo alternativo que considero más promisorio. Revisaré en particular el modo en el que podemos capturar formalmente el concepto de conocimiento común. En la Sección 3 examinaré algunos argumentos de Philip Pettit y Christian List (incluyendo su análisis del llamado "dilema discursivo"), que nos llevarán a afirmar que, al menos en algunas oportunidades, el conocimiento grupal podría entenderse técnicamente como conocimiento distribuido. En la Sección 4 haré explícitas algunas consecuencias de esta afirmación; en particular, mostraré que el concepto de conocimiento distribuido impone un nuevo desiderátum sobre los agentes ideales. En la Sección 5 mostraré como conciliar intuiciones encontradas a través de un marco dinámico, deudor de las llamadas "Logics of Public Announcements", que permita identificar secuencias de modelos a lo largo del tiempo. Sobre la base de estas ideas, en la Sección 6 daré una formulación apropiada para el concepto de conocimiento grupal, que será entendido no como un conjunto de proposiciones, sino más bien como una expectativa de apropiación de dichas proposiciones por parte de los miembros del grupo.

Cf. FAGIN, Ronald; HALPERN, Joseph Y.; MOSES, Yoram; VARDI, Moshe Y., Reasoning About Knowledge, Cambridge, Mass.: MIT Press, 1995. 
En la última sección ofreceré algunas conclusiones, así como respuestas a algunas posibles objeciones.

\section{Transparencia epistémica y responsabilidad individual}

Tal como he anticipado, comenzaré por mencionar brevemente un proyecto anterior sobre transparencia epistémica, responsabilidad epistémica y conocimiento individual, y por explicar por qué me parece relevante continuarlo del modo en que será continuado en estas páginas. La transparencia epistémica nos dice que si un sujeto sabe que $p$, entonces sabe que sabe que $p$. En lógica modal se lo suele formular como un axioma que regula el funcionamiento del operador $K$ de conocimiento:

$$
\vDash K p \rightarrow K K p \quad \text { para toda proposición } p
$$

Ha sido llamado principio de reflexividad del conocimiento, principio de introspección positiva, de auto-conocimiento, de transparencia o de luminosidad epistémica; me referiré a él indistintamente con cualquiera de estos nombres. En trabajos anteriores ${ }^{2}$ argumenté a favor de la adopción de una versión moderada de transparencia (es decir, una versión debilitada del condicional señalado más arriba), como principio normativo, por razones de coherencia formal.

Ahora bien, ¿por qué querríamos defender la transparencia epistémica? ¿Por qué nos importa? La explicación es que la transparencia epistémica constituye un ideal de la responsabilidad epistémica, y en última instancia, del ser plenamente agentes, aunque no necesariamente de la racionalidad ideal. En este punto es importante reconocer que la responsabilidad epistémica viene en grados; según una idea robusta de responsabilidad, nos resistimos a decir que un agente es plenamente responsable de su creencia de que $p$ (digamos, de su creencia de que Madrid es la capital de España, de que en este momento hace calor en Buenos Aires, de que cierto tipo de políticas fomentan la fuga de divisas, etc.), a menos que el agente sea consciente de sostener que $p$, y persevere en su creencia luego de reflexionar sobre ella. Al juzgar que $S$ es plenamente responsable en relación con dicha creencia, no solamente percibimos a $S$ como alguien capaz de defender que $p$ (eso se aplica a cualquiera que tenga la creencia en cuestión) sino que lo percibimos como alguien que se percibe a sí mismo como un defensor de $p$ : alguien que sabe exactamente lo que está

2 Cf. CRESTO, Eleonora, A Defense of Temperate Epistemic Transparency, in: Journal of Philosophical Logic, 41:6 (2012), p. 923-955. 
haciendo cuando defiende que $p$. Juzgarlo responsable es, digamos, obligarlo a hacerse cargo de sus ideas; es ubicarlo en el lugar de quien puede decir con verdad: "yo soy, entre otras cosas, alguien que defiende que $p$ en este momento" - y es esperar que actúe en consecuencia. La posibilidad de que los agentes a veces abracen creencias en este sentido pleno captura algo extremadamente importante de la idea que tenemos de nosotros mismos en tanto personas. Entonces, este sentido de responsabilidad es claramente un desideratum. Para decirlo en forma de slogan: "asegúrate de ser dueño de tus propias creencias" (y deseos).

La transparencia, entonces, bajo alguna formulación adecuada, es un requisito de la responsabilidad plenamente idealizada de los agentes individuales, con lo cual el siguiente condicional resulta conceptualmente válido: si $S$ es un agente responsable plenamente idealizado y $S$ sabe que $p$, entonces $S$ ha reflexionado debidamente sobre sus estados epistémicos de primer orden, y ha encontrado que es el caso que sabe que $p$.

Hasta aquí la discusión se refirió a la suerte de agentes individuales. Pero hay entidades colectivas que también pueden concebirse como agentes, puesto que podemos atribuirles estados intencionales, como deseos y creencias. Se trata en este caso de agentes grupales, como instituciones, clubes, o grupos de estudio. ¿Puede extenderse el análisis anterior a los agentes colectivos, a los grupos? Dado el papel central que desempeñan los grupos de diversa índole en la vida de cada uno de nosotros, me parece importante examinar esta pregunta.

En particular, la idea de que la reflexividad epistémica es una condición necesaria de la responsabilidad vale también para los agentes grupales. La razón es simple. En la medida en que percibimos a un grupo como un agente colectivo, y no como un mero agregado, lo concebimos como sujeto de propiedades características de los agentes. Y para hablar de agencia parece fundamental no solamente la posibilidad de atribuir actitudes proposicionales, sino, concomitantemente, la posibilidad de atribuir responsabilidad, tanto epistémica como moral. Las mismas consideraciones que nos llevaron en su momento a afirmar que la responsabilidad individual es cuestión de grado se aplican aquí: también para el caso grupal podemos hablar de responsabilidad más o menos idealizada. Sugiero que, tal como ocurría en el caso individual, la responsabilidad epistémica grupal ideal necesita que el agente en cuestión (que aquí es un agente colectivo) sepa que cuenta con las creencias que de hecho tiene, y que de este modo las ratifique mediante una actitud grupal apropiada de segundo orden.

¿Habrá aquí también razones formales (además de filosóficas, o conceptuales) para pedir transparencia o reflexividad epistémica a nivel de los agentes grupales? Entiendo que la respuesta es afirmativa. Esto 
es, es posible mostrar que obtenemos reflexividad en el conocimiento grupal no solamente por razones filosóficas relacionadas con el pedido de responsabilidad, sino con razones formales.

Sin embargo, en el caso grupal que nos interesa ahora será necesario prestar especial atención al modo en que interactúan conocimiento y responsabilidad grupales con sus contrapartes a nivel individual. La tesis central de este trabajo es que el desideratum de responsabilidad grupal impone inmediatamente nuevos desiderata sobre los individuos idealmente responsables. $Y$ esto, en última instancia, nos revela la naturaleza esencialmente inestable (o, si se prefiere, dinámica) de los agentes grupales.

\section{Un marco formal para razonar sobre el conocimiento de agentes grupales. El concepto de conocimiento común}

Empecemos recordando una definición clásica de creencia grupal, que ha resultado sumamente influyente en las discusiones de epistemología social de las últimas décadas. Leemos en Tuomela (1992), por ejemplo:

$G$ cree que $p$ en las circunstancias sociales y normativas $C$ si y sólo si hay en $C$ miembros operativos $A_{1} \ldots A_{m}$ de $G$ respectivamente en las posiciones $P_{1} \ldots P_{m}$ tal que:

(1) Los agentes $A_{1} \ldots A_{m}$, cuando llevan a cabo sus tareas sociales en las posiciones $P_{1} \ldots P_{m}$ y debido al ejercicio del sistema de autoridad relevante de $G$, (intencionalmente) conjuntamente aceptan que $p$ como la visión de $G$, y gracias a este ejercicio del sistema de autoridad, deben continuar aceptándolo y creyéndolo posicionalmente;

(2) Hay una creencia mutua entre los miembros operativos $A_{1} \ldots A_{m}$ acerca de que (1);

(3) Gracias a (1), los miembros no operativos (plenos y adecuadamente informados) de $\mathrm{G}$ tienden tácitamente a aceptar - o por lo menos deberían aceptar - que $p$, como miembros de G; y

(4) Existe una creencia mutua en $G$ acerca de que (3) ${ }^{3}$.

Antes de continuar es necesario hacer un par de aclaraciones importantes. Primero, uno de los temas que le interesan a autores como Tuomela es la diferencia entre creencia individual y aceptación (como un tipo de actitud proposicional que no necesariamente apunta a la verdad). Aquí no me ocuparé especialmente de este problema; en otras palabras,

3 Cf. TUOMELA, Raimo, Group Beliefs, in: Synthese, 91 (1992), p. 295-296, traducción y énfasis mío. Cf. también GILBERT, Margaret, On Social Facts, London - New York: Routledge, 1989, entre otros. 
no discutiré si las actitudes individuales relevantes que se encuentran a la base del concepto de creencia grupal son actitudes intencionales de creencia propiamente dichas o de mera aceptación. Todo lo que se diga en lo sucesivo sobre actitudes doxásticas individuales puede traducirse, si así se desea, en términos de 'aceptaciones'.

Segundo, y relacionado con lo anterior, la cita de Tuomela no nos dice nada sobre las posibles actitudes epistémicas del grupo. Como es sabido, la agregación de actitudes doxásticas constituye un problema especialmente complicado justamente porque, como las creencias (o aceptaciones) no necesitan ser verdaderas, diferentes agentes pueden tener conjuntos mutuamente inconsistentes. No ocurre algo análogo con el conocimiento. En esta oportunidad no me ocuparé del problema más general de la agregación de creencias. A pesar de todo ello, desde el momento en que el conocimiento grupal es un tipo de creencia grupal, algunas de las moralejas que podamos extraer de las discusiones sobre creencia grupal serán relevantes también para el conocimiento. En cualquier caso, es importante aclarar que el concepto de conocimiento que nos ocupará aquí podría entenderse simplemente como 'información', o 'creencia verdadera'; en este trabajo no pondré ningún énfasis en capturar la noción tradicional de justificación.

Volvamos a la cita de Tuomela. Allí se pide que sean los llamados 'miembros operativos' del grupo quienes acepten [crean] que $p$; para el resto de los miembros se pide algo más débil, el equivalente de una aceptación [o creencia] tácita. Nuevamente, no voy a entrar en detalles sobre esta importante distinción. Lo que sí quiero destacar es que de la definición anterior se desprende que una creencia grupal no puede ser meramente una actitud que comparten todos los miembros, ni siquiera un subconjunto privilegiado de ellos; a fortiori, esto mismo ha de valer para una creencia que constituya conocimiento grupal: se requiere creencia mutua sobre dichos compromisos de primer orden, así como creencia mutua sobre la aceptación tácita de los miembros no operativos; obsérvese que aquí Tuomela bien podría haber pedido conocimiento mutuo, o, en otra terminología, conocimiento común sobre dichas actitudes de primer orden, y no habría ninguna diferencia sustancial en el espíritu de su propuesta. Es sabido que se trata de una exigencia muy fuerte, que va mucho más allá de la mera demanda de conocimiento compartido (o, mutatis mutandis, creencia compartida).

Antes de continuar es conveniente fijar la terminología, para evitar malos entendidos; en lo que sigue me baso de manera crucial en Fagin et alii (1995) ${ }^{4}$. Consideremos un lenguaje proposicional $L_{E}$ enriquecido

4 Cf. FAGIN, Ronald; HALPERN, Joseph Y.; MOSES, Yoram; VARDI, Moshe Y., op. cit., 1995. 
con operadores epistémicos para $n$ agentes diferentes, en el modo usual, de modo que podamos expresar:

(i) El agente $i$ sabe que $f: K_{i} \phi$

(ii) Los agentes $1 \ldots n$ saben que f: $E_{\{1 \ldots n\}} \phi$

(iii) $E^{k+1} f={ }_{\text {def }} E E^{k} \phi$

(iv) El grupo compuesto por los agentes 1...n tiene conocimiento común de que $f$ : $C_{\{1 \ldots n\}} f$, donde $C_{\{1 \ldots n\}} f=\grave{\mathrm{U}}^{\infty}{ }_{k=0} E^{k} \phi$

Donde $i$ es cualquier agente, y $f$ es una oración cualquiera del lenguaje.

' $E_{\{1, \ldots n\}} f^{\prime}$, para cualquier oración $f$, es la afirmación de que los agentes $1 \ldots n$ tienen conocimiento compartido de que $f$. Cuando se sobreentienda de qué agentes estamos hablando abreviaré la expresión anterior simplemente como ' $E f$ '. A falta de un rótulo mejor, llamémoslo 'conocimiento compartido' de que $f$; todos en el grupo $\{1 \ldots n\}$ saben que $f$. De manera análoga podríamos definir la idea de 'creencia compartida'. Desde luego, los agentes pueden tener conocimiento compartido del hecho de que tienen conocimiento compartido, como en (iii). Finalmente, sea " $C_{\{1 \ldots n\}}$ " la proposición que dice "el grupo formado por los individuos 1 ...n tiene conocimiento común de que $f^{\prime}$ "; nuevamente, lo abreviaremos como ' $\mathrm{Cf}$ ' cuando no haya dudas sobre qué grupo se trata. Esto significa que no solamente los agentes saben que $f$, sino que saben que saben que f; también saben que saben que saben que f, y así infinitamente; el conocimiento compartido aquí ha sido infinitamente iterado.

La explicación semántica de estos conceptos en un marco kripkeano también es bien conocida. Consideremos una estructura $M=<W$, $R_{1}, \ldots, R_{n} \ldots, v>$, en la que $W$ es un conjunto de mundos posibles, $R_{1}, \ldots, R_{n}$ son relaciones de accesibilidad entre mundos (una para cada agente), y $v$ es una función de valuación. Como es usual, supondré que las proposiciones son conjuntos de mundos posibles. Como en cualquier modelo kripkeano, las relaciones de accesibilidad regulan el comportamiento de los operadores $K_{i}$ de conocimiento, para cada agente $i^{5}$. Además, para cada agente $i$ y mundo $w$, sea $R_{i}(w)$ la proposición más fuerte conocida por $i$ en $w$ (es decir que dicho conjunto de mundos estará incluido en cualquier otra proposición sabida por $i$ ); esto es, para todo agente $i$ y mundo $w$ :

\footnotetext{
In: CRESTO, Eleonora, A Defense of Temperate Epistemic Transparency, op. cit., p. 923-955, sugiero considerar una secuencia de relaciones de accesibilidad para cada agente, ya que allí presupongo que existe una jerarquía de operadores de conocimiento distintos para órdenes superiores. El modelo se complementa luego con secuencias de funciones de probabilidad evidencial. En esta oportunidad voy a prescindir de dichas herramientas, por razones de simplicidad. Las conclusiones del presente trabajo podrán luego refinarse adecuadamente, en instancias posteriores.
} 


$$
R_{i}(w)=\left\{w^{\prime}: w R_{i} w^{\prime}\right\}
$$

Así, si una oración y expresa exactamente la proposición ' $R_{i}(w)$ ', entonces desde luego el agente sabe que y en $w$, i.e.: sea $R_{i}(w)=\left\{w^{\prime}\right.$ ': $\left.M, w^{\prime} \vDash \psi\right\}$; entonces $M, w \vDash K_{i} \psi$

Para hacer la discusión un poco más concreta, supongamos un modelo particular con cinco mundos (w1...w5) y dos agentes (agentes 1 y 2 ), como se muestra en el diagrama de más abajo. Aquí ambas relaciones $R_{1}$ y $R_{2}$ son reflexivas y no transitivas:

El conjunto más fuerte (más pequeño) que representa todo lo que el agente 1 sabe en $w 1$, o $R_{1}(w 1)$, es $\{w 1, w 2\}$. El conjunto más fuerte que representa todo lo que el agente 2 sabe en $w 1$, o $R_{2}(w 1)$, es, evidentemente, $\{w 1, w 3\}$. ' $E \mathrm{f}$ ' será verdadero en un mundo cualquiera si en ese mundo todos los agentes saben que f. Por ejemplo, la disyunción entre $R_{1}(w 1)$ y $R_{2}(w 1)$ es conocimiento compartido en $w 1$; es decir, obtenemos que

$$
M, w 1 \vDash E\left(R_{1}(w 1) \vee R_{2}(w 1)\right) .
$$

Desde un punto de vista semántico, la relación de accesibilidad que caracteriza al conocimiento común es la clausura transitiva de la unión de todas las relaciones de accesibilidad de los agentes individuales ${ }^{6}$. En nuestro ejemplo, la relación en cuestión contendrá todos los pares ordenados que teníamos antes, más el par (w1, w4). A la relación de accesibilidad que gobierna al operador $C$ la llamaremos $R C$; correspondientemente, $R C_{\{1,2\}}(w i)$, para algún $i$, será el conjunto más fuerte que representa todo lo que el grupo $\{1,2\}$ conoce en $w_{i}$ :

$$
R C(w)=\left\{w^{\prime}: w R C w^{\prime}\right\}
$$

$$
\text { Así, } R C_{\{1,2\}}(w 1)=\{w 1, w 2, w 3, w 4\}^{7} \text {. }
$$

6 Cf. FAGIN, Ronald; HALPERN, Joseph Y.; MOSES, Yoram; VARDI, Moshe Y., op. cit., 1995.

7 Podríamos acuñar también el concepto de 'creencia común', o ' $C_{B}$ ', con las mismas características formales. Desde luego, $C_{B}$ deberá basarse en relaciones de accesibilidad que regulen apropiadamente el comportamiento del operador $B$ de creencia, y no el operador $K$; obsérvese que éstas no tienen por qué ser reflexivas, ya que $B$ no tiene por qué verificar el llamado axioma $T$ (' $B p \rightarrow p$ ' no es en general una oración verdadera en todo mundo). La pregunta de cómo construir un modelo combinado satisfactorio no es trivial; para algunas discusiones al respecto cf. DE BRUIN, Boudewijn, Epistemic Logic and Epistemology, in: HENDRICKS, Vincent and PRITCHARD, Duncan (eds.), New Waves in Epistemology, New York: Palgrave Macmillan, 2008, p. 106-136. Un requisito mínimo para combinar ambos operadores es que, para cada agente, la relación de accesibilidad para $B$ esté incluida en la correspondiente relación para $K$, de modo que, para cualquier proposición $p$, se valide la fórmula ' $K p \rightarrow B p$ ' (ya que el conocimiento implica creencia). En este trabajo no intentaré dar respuesta a estas preguntas. 


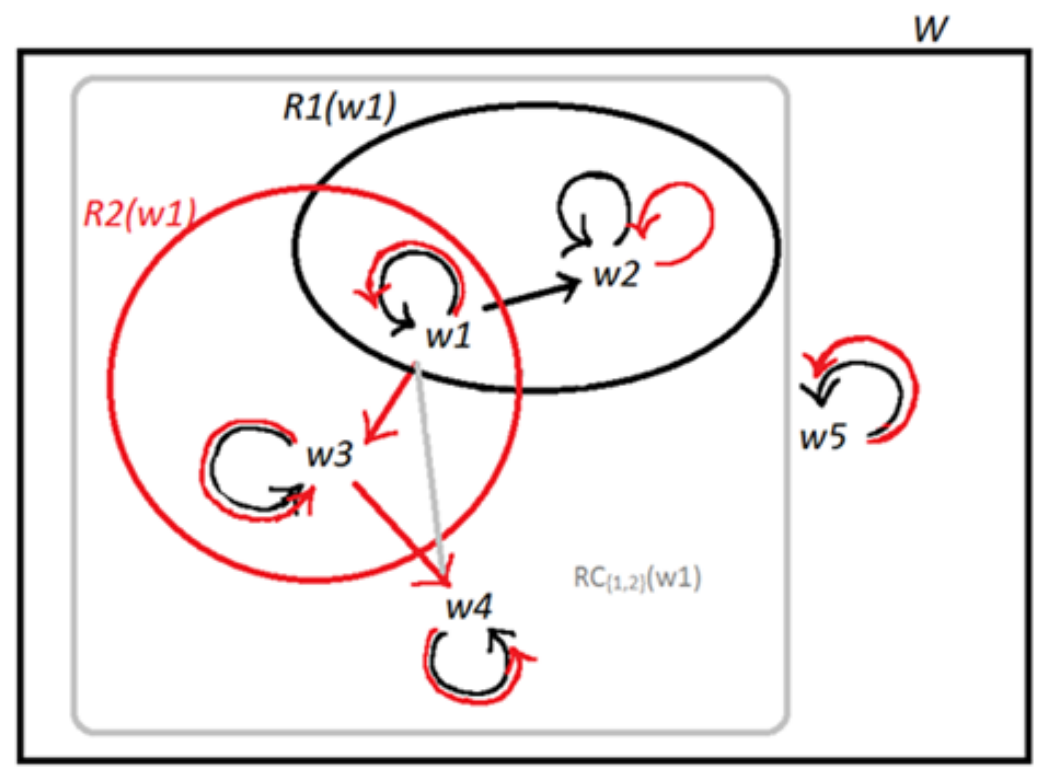

Para recapitular: digamos que $\operatorname{Know}_{i}(w)$ representa lo que el agente $i$ sabe en $w$, para algún $w$ en $M$ (en un tiempo dado). Entonces puede suponerse que el conocimiento que el agente $i$ tiene en $w$ queda representado por un conjunto particular de proposiciones: aquella que contienen $R_{i}(w)$ :

$$
\operatorname{Know}_{i}(w)=\left\{\mathrm{x} \in 2^{W}: R_{i}(w) \subseteq \mathrm{x}\right\}
$$

Por otro lado, digamos que $\operatorname{Know}_{G}(w)$ representa lo que el grupo sabe en $w$, para algún $w$ y $M$ (en un tiempo dado). Si el conocimiento grupal es interpretado como conocimiento común (lo cual aún está por verse que sea una buena idea), entonces, en $w$, el grupo conoce todas las proposiciones que contengan $R C(w)$ :

$$
(G C K) \quad \operatorname{Know}_{G}(w)=\left\{\mathrm{x} \in 2^{W}: R C(w) \subseteq \mathrm{x}\right\}
$$

Así la cosas, notemos lo siguiente:

En primer lugar, es importante mencionar que el conocimiento común (y, de modo análogo, la creencia común) satisface transparencia, independientemente de cómo sean las relaciones de accesibilidad $R_{i}$ de cada individuo. Es decir que, para toda oración $\phi$, todo mundo $w$ y todo modelo, tenemos que: 
Este es un resultado más o menos inmediato de la manera en que procede la semántica para el operador $C$, ya sea de creencia o de conocimiento. Hay un sentido, pues, en el que podríamos decir que tanto el conocimiento como la creencia común funcionan como una suerte de autoconciencia grupal, garantizando así la responsabilidad epistémica del colectivo. Y esto no sólo porque es posible obtener conocimiento (y creencia) común de segundo orden de manera automática, sino en verdad por la naturaleza misma ('cuasi-hegeliana', para decirlo un tanto metafóricamente) del fenómeno en cuestión, en el cual cada agente individual se ve involucrado en un proceso de reconocimiento infinito sobre las actitudes epistémicas de los otros individuos. Obsérvese que el conocimiento común del grupo en un mundo es siempre igual o más débil que el conocimiento que tiene en ese mundo cada uno de los individuos.

En segundo lugar, obsérvese que las siguientes fórmulas son válidas, en todo modelo:

$$
\begin{aligned}
& \vDash C E \phi \rightarrow C \phi \\
& \vDash C K_{i} \phi \rightarrow C \phi
\end{aligned}
$$

para todo $i$ y todo $\phi$.

La moraleja es que, toda vez que pidamos conocimiento común sobre otras actitudes proposicionales, tendremos inmediatamente conocimiento común sobre los objetos de las actitudes de primer orden. Otra manera de decirlo podría ser: la autoconciencia grupal no puede limitarse a otras actitudes de primer orden. Si nos comprometemos con la idea de que todo auténtico conocimiento grupal requiere de autoconciencia grupal, entonces todo conocimiento grupal pasa a ser conocimiento común. No hay intermedios.

Esto es desde luego trivial, formalmente hablando, pero puede ser filosóficamente relevante: entre otras cosas, nos dice que no podemos tener un análogo del análisis de Tuomela para el conocimiento: las sutilezas ya mencionadas en la cita de Tuomela, en el sentido de que diferentes miembros del grupo pueden estar a cargo de diferentes actitudes, dejan de ser posibles una vez que requerimos que haya conocimiento común sobre esas mismas actitudes ${ }^{8}$.

8 Algunas palabras de cautela. Tuomela en verdad pide que haya conocimiento común (o creencia común) entre los miembros operativos, acerca de que dichos miembros creen (o aceptan) que $\phi$, así como conocimiento común (o creencia común) entre todos los miembros del grupo acerca de que los miembros no operativos tienden tácitamente a creer (o aceptar) que $\phi$. Un análogo de este análisis para el conocimiento, presumiblemente, pediría que hubiera conocimiento común entre los miembros operativos acerca del hecho de que dichos 


\section{El conocimiento grupal como conocimiento distribuido}

A esta altura alguien podría estar tentado de aceptar las consecuencias indeseables y decir que el conocimiento grupal simplemente es conocimiento común, sin lugar para sutilezas. Pero no es difícil advertir que tenemos intuiciones respecto del conocimiento grupal que van exactamente en dirección opuesta. Para ver esto, recordemos aquí el llamado 'dilema discursivo', explorado en los últimos años sobre todo a partir de algunos trabajos de Philip Pettit y Christian List ${ }^{9}$. Supongamos que en un grupo formado por tres agentes se consideran por turno las opiniones individuales respecto de diversas cuestiones, en todas las cuales ha de decidirse por sí o por no, y supongamos que todos acuerdan en que se tomará como opinión del grupo, en todos los casos, la opinión de la mayoría. Así las cosas, bien podría ocurrir que el grupo termine sosteniendo actitudes proposicionales inconsistentes. En el ejemplo que se ilustra más abajo, la mayoría vota por $A$ y por $A \rightarrow B$, pero en contra de $B$ : si bien las actitudes de cada uno de los agentes individuales son internamente consistentes, la del grupo no lo es.

\begin{tabular}{|c|c|c|c|}
\hline & A & $\mathbf{A} \rightarrow \mathbf{B}$ & $\mathbf{B}$ \\
\hline Agente (1) & Sí & No & No \\
\hline Agente (2) & No & Sí & No \\
\hline Agente (3) & Sí & Sí & Sí \\
& Sí & Sí & No \\
\hline
\end{tabular}

miembros saben que $\phi$, y pediría además que hubiera conocimiento común entre todos los miembros del grupo acerca de que los miembros no operativos saben tácitamente que $\phi$. Esta última es justamente la afirmación problemática: no puede haber en $G$ conocimiento común de que, digamos, $G^{\prime}$ sabe tácitamente que $\phi$ sin que al mismo tiempo haya conocimiento común en $G$ de que $\phi$. Podríamos frenar esta inferencia, sin embargo, si adoptáramos un sentido peculiar de 'saber tácitamente'. Pero no es claro que tengamos razones para hacerlo. Al menos pre-teóricamente, pareciera que si María sabe que Pedro sabe tácitamente que $\phi$, entonces María sabe que $\phi$ (tal como ocurre con el concepto regular de saber). Para evitar este resultado podríamos argumentar que el concepto regular de conocimiento debe entenderse como conocimiento explícito, de modo tal que si $S$ sabe tácitamente que $\phi$, entonces $S$ no sabe (explícitamente) que $\phi$. Pero entonces decir "María sabe explícitamente que ella sabe tácitamente que $\phi$ " (análogamente, afirmar que hay conocimiento común en el grupo de María acerca del hecho de que ella sabe tácitamente que $\phi$ ) está al borde de la contradicción; es como decir "María es consciente del hecho de que no es consciente del hecho de que $\phi$ ".

9 Cf. entre otros PETTIT, Philip, Groups with Minds of Their Own, in: SCHMITT, Frederick (ed.), Socializing Metaphysics, New York: Rowman and Littlefield, 2003, p. 167-193, y LIST, Christian and PETTIT, Phillip, Group Agency: The Possibility, Design, and Status of Corporate Agents, Oxford: Oxford University Press, 2011; para una perspectiva crítica, cf. LACKEY, Jennifer, Group Belief: Lessons from Lies and Bullshit, in: IDEM, The Epistemology of Groups, Oxford: Oxford University Press, en prensa. La paradoja comienza a ser discutida en los años ochenta, en el ámbito del Derecho; cf. KORNHAUSER, L. A. y SAGER, L. G., "Unpacking the Court", in: Yale Law Journal, 96 (1986), p. 82-117. 
Una manera posible de restablecer la consistencia, con la que yo personalmente simpatizo, consiste en aceptar que a veces los grupos forman creencias simplemente sobre la base de premisas previamente consensuadas; ello permite, como caso límite, que haya creencias grupales que no sean a su vez la creencia de ningún individuo del grupo:

\begin{tabular}{|c|c|c|c|c|}
\hline & A & B & C & $\mathbf{A} \wedge \mathbf{B} \wedge \mathbf{C}$ \\
\hline Agent (1) & Yes & No & Yes & No \\
\hline Agent (2) & No & Yes & No & No \\
\hline Agent (3) & No & Yes & Yes & No \\
Agent (4) & Yes & No & Yes & No \\
Agent (5) & Yes & Yes & No & No \\
& Yes & Yes & Yes & No \\
\hline
\end{tabular}

Pettit va aún más lejos y afirma ${ }^{10}$ que los grupos que sistemáticamente se privan de la posibilidad de 'colectivizar la razón' y formar conclusiones sobre la base de premisas ya consensuadas, no son propiamente grupos, en el sentido más rico de este término (como diferente de, por ejemplo, meros agregados de individuos). En el resto del trabajo daré por sentado, sin más argumentos, que esta afirmación es correcta. Dicho de otro modo, el interlocutor pretendido de este trabajo es alguien que ha aceptado el punto de vista general de Pettit, aunque tal vez no haya reflexionado suficientemente sobre sus consecuencias.

Como ya he mencionado, no me ocuparé aquí del problema general de la agregación de creencias. Y notemos que no existe un dilema equivalente para el conocimiento, dado que dos agentes diferentes no pueden conocer proposiciones incompatibles. Sin embargo, una de las enseñanzas fundamentales que nos deja el dilema discursivo resulta apropiada para nuestros fines. La moraleja es que, si queremos hablar de actitudes grupales, no podemos dejar de 'colectivizar la razón': no podemos prescindir sistemáticamente de procedimientos inferenciales: al menos a veces debemos poder atribuirle a los grupos actitudes que no pertenecen a ninguno de sus miembros (de otro modo ponemos en peligro la agencia grupal). Si las actitudes en cuestión son actitudes epistémicas, esta línea de razonamiento, que favorece procedimientos de agregación basados en la inferencia a partir de premisas ya consensuadas (premise-based procedures) da apoyo a una manera particular de entender el conocimiento grupal: a saber, como conocimiento distribuido.

10 Cf. PETTIT, Philip, Groups with Minds of Their Own, in: SCHMITT, Frederick (ed.), op. cit., p. 167-193. 
Siguiendo nuevamente a Fagin et alii $(1995)^{11}$, diremos que en un mundo dado $w$ un grupo de individuos tiene conocimiento distribuido de que $f$ (lo que abreviaremos ' $D f$ ') si la intersección de todas las relaciones de accesibilidad de los agentes nos deja llegar sólo a mundos- $f$ desde $w$. A la relación de accesibilidad que gobierna el operador $D$ la llamaremos $R D$. Así,

$$
R D(w)=\left\{w^{\prime}: w R D w^{\prime}\right\}
$$

Si retomamos el ejemplo sencillo de la sección anterior, notaremos que el conocimiento distribuido más fuerte que tiene el grupo en un mundo w1 es el conjunto más pequeño de mundos que se relacionan con $w 1$ gracias a $R D$. A diferencia de lo que pasa en el caso de conocimiento común, el conocimiento distribuido siempre es igual o más fuerte para el grupo que para los individuos (o sea, $R D(w 1)$ es siempre igual o más pequeño que $R_{1}(w 1)$ y $\left.R_{2}(w 1)\right)$.

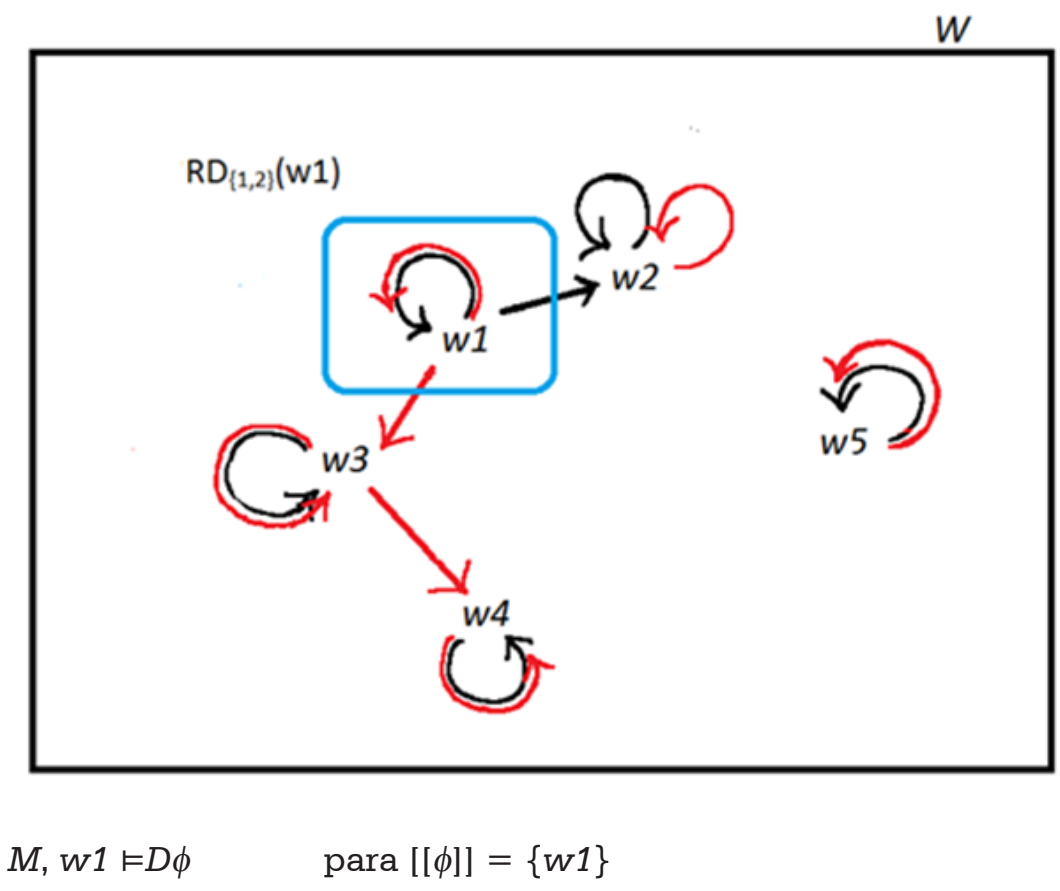

¿Qué hemos de entender por una instancia de conocimiento grupal, pues? Por el momento, todo lo que hemos mostrado es que, al menos

${ }_{11}$ Cf. FAGIN, Ronald; HALPERN, Joseph Y.; MOSES, Yoram; VARDI, Moshe Y., op. cit., 1995. 
algunas veces, en algunas circunstancias, nuestra comprensión preteórica de qué sea el conocimiento grupal se acerca notablemente al concepto de conocimiento distribuido. Lo cual equivale a decir que el conocimiento grupal en $w$ algunas veces debería capturarse como el conjunto de proposiciones que incluyen $R D(w)$ :

$(G D K)$

$$
\operatorname{Know}_{G}(w)=\left\{\mathrm{x} \in 2^{W}: R D(w) \subseteq \mathrm{x}\right\} \quad \text { (para un } w \text { y } M \text { dados) }
$$

En otras palabras, tal vez no todo conocimiento grupal sea conocimiento distribuido, ni toda creencia grupal sea creencia distribuida, pero al menos algunas instancias sí lo serán. Vale la pena discutir algunas consecuencias importantes de esta afirmación ${ }^{12}$. Lo haremos en la próxima sección.

\section{Responsabilidad epistémica y conocimiento distribuido: algunos problemas}

Consideremos en primer lugar la siguiente definición. Diré que una instancia de conocimiento distribuido es no trivial si y sólo si ninguno de los agentes individuales comparte el conocimiento en cuestión. Es sencillo ver que no puede haber instancias no triviales de conocimiento común sobre conocimiento distribuido, dado que los siguientes condicionales son válidos (para cualquier $f$ y cualquier agente $i$ ):

$$
\begin{aligned}
& \vDash K_{i} D \phi \rightarrow K_{i} \phi \\
& \vDash C D \phi \rightarrow C \phi
\end{aligned}
$$

Este resultado no es sino una nueva consecuencia de nuestras consideraciones previas sobre el comportamiento formal del operador $C$. Nuevamente, esto es formalmente trivial, pero nos alerta sobre el hecho de que muy probablemente tengamos intuiciones encontradas, mutuamente insatisfacibles, sobre la manera de concebir a los agentes grupales y sus actitudes intencionales. Por un lado, para poder hablar de agencia grupal parece razonable pedir que exista algún tipo de reflexión conjunta sobre el saber del grupo, como quería Tuomela; pero por otro lado, parece razonable también pedir que la entidad en cuestión sea capaz de 'colectivizar la razón'. Y no podemos tener ambas cosas a la vez. Un poco más adelante intentaremos alguna manera de resolver esta tensión.

12 Desde un punto de vista formal, podríamos también intentar caracterizar al conocimiento grupal mediante una nueva relación de accesibilidad sui generis (debo esta sugerencia a Elia Zardini). El problema es que, de proceder de este modo, estaríamos abandonando el individualismo metodológico; el difícil entender qué constreñiría una relación de este tipo. 
Veamos ahora una segunda consecuencia interesante, aún más seria. Supongamos que los agentes individuales que componen el grupo son todos ellos idealmente responsables. Es decir que vale el principio $K K$, para todo agente:

$$
\vDash K_{i} \phi \rightarrow K_{i} K_{i} \phi \quad \text { para todo } \phi
$$

Como es sabido, ello significa que las relaciones de accesibilidad de los agentes son transitivas ${ }^{13}$. En este caso es sencillo mostrar que el conocimiento distribuido también satisface transparencia. En otras palabras, es cierto para toda proposición $p$, en todo mundo posible, que

$$
\vDash D \phi \rightarrow D D \phi^{14}
$$

El hecho de que para individuos idealmente responsables tanto $C$ como $D$ sean reflexivos reviste una enorme importancia conceptual. Al menos en los casos típicos, nadie cuestiona que los individuos sean agentes; en general, aquellos autores que niegan la validez del Principio $K K$ a lo sumo querrán comprometerse con la idea de que los agentes reales no siempre son idealmente responsables ${ }^{15}$. Pero en el caso grupal la situación es más complicada; si hubiera obstáculos formales más o menos evidentes para la reflexividad epistémica o doxástica, ello favorecería inmediatamente una mirada escéptica sobre la agencia grupal ${ }^{16}$. En síntesis, constatar que, cuando los individuos son responsables, tanto $D$ como $C$ son operadores reflexivos elimina un obstáculo importante para

13 En un marco más sofisticado que permita jerarquías de operadores epistémicos diferentes, los agentes individuales idealmente responsables validarán una versión moderada del principio $K K$, a saber, ' $K^{1} \phi \rightarrow K^{2} K^{1} f^{\prime}$ (y, más generalmente, ' $K^{i} \phi \rightarrow K^{i+1} K f^{\prime}$ ); se puede probar que dichos principios no requieren que las relaciones de accesibilidad sean transitivas (cf. CRESTO, Eleonora, A Defense of Temperate Epistemic Transparency, op. cit., p. 923-955).

14 En el caso del conocimiento común, la transitividad está garantizada por definición, independientemente de cómo sean las relaciones de accesibilidad individuales. En el marco alternativo sugerido en la nota anterior, la reflexividad del operador $D$ es también automática, independientemente de cómo sea $R_{i}$ en el nivel 1, para cualquier $i \in\{a \ldots n\}$.

15 Desde luego, la mayoría de dichos autores no tiene presente la posible conexión entre conocimiento de segundo orden y responsabilidad; el rechazo a $K K$ suele estar motivado por consideraciones empíricas acerca de cómo conocen los agentes reales.

16 Nótese que el operador $E$ no es igualmente reflexivo por razones semánticas. Dicho de otro modo, la transitividad de las $R_{i}$ no garantiza la reflexividad de $E$. Así, aún si los agentes son individualmente responsables, de lo que meramente saben todos, el grupo, qua grupo, no necesita ser epistémicamente responsable. Por supuesto, del hecho de que $E$ no sea reflexivo por razones semánticas no se sigue que no pueda satisfacerse empíricamente que $E p \rightarrow E E p$, para un grupo dado. En cualquier caso, recuérdese que, según el análisis de las secciones anteriores, pedimos bastante más que $E$ para hablar de creencia o conocimiento grupal; en verdad, uno podría tomar el hecho de que $D$ y $C$ permiten hablar de responsabilidad mientras que $E$ no lo hace (o al menos, no lo hace necesariamente), como evidencia de que $E$ no puede capturar una actitud grupal. Aquí no voy a perseverar en esta línea argumentativa, pero vale la pena explorarla. 
afirmar la posibilidad de responsabilidad estrictamente grupal, y con ella la posibilidad de agencia. De modo que aquellos que vemos con buenos ojos la idea de la existencia de agencia grupal deberíamos saludar la reflexividad de $D$ como buenas noticias.

Sin embargo, las cosas no son tan sencillas. Lo cierto es que, en el caso del conocimiento o creencia distribuidos, la mentada 'autoconciencia' grupal actúa de un modo muy singular, de un modo anormal, que tendremos que examinar con cierto cuidado.

Ciertamente, hemos obtenido que si un grupo de individuos responsables tiene conocimiento distribuido de que $\phi$, ello quiere decir que el grupo tiene la autoconciencia necesaria para 'ratificar' que $f$ bajo reflexión. Sin embargo, este sentido de reflexión se aleja bastante de nuestra intuición de qué es reflexionar, ya que bien podría ocurrir que ningún individuo concreto haya de hecho reflexionado de este modo. En otras palabras, la reflexividad del conocimiento distribuido implica que es el grupo, qua grupo, el que en algún sentido se ha pensado a sí mismo y ha generado actitudes proposicionales de segundo orden.

Se dirá que eso era justamente lo que buscábamos: independizar la intencionalidad grupal de la individual. Sin embargo, podríamos preguntarnos si tal independencia sigue siendo razonable para las actitudes proposicionales de segundo orden. ¿Es la intencionalidad grupal de segundo orden realmente independiente de la intencionalidad individual de segundo orden? Veremos que aceptar la independencia de actitudes epistémicas grupales de segundo orden parece bastante más oneroso, ontológicamente hablando, que aceptar las correspondientes actitudes de primer orden sin contraparte individual; parece producir un tipo de inflación ontológica más profunda que la que veníamos tolerando hasta el momento.

Consideremos la diferencia entre:

(A) "El Hospital sabe perfectamente bien que la mitad de los residentes se han negado a responder preguntar sobre su estado civil."

(B) "El informe que tengo en mis manos ofrece una descripción detallada de todo lo que el Partido sabía en aquel momento acerca de este desafortunado incidente."

(C) "La Junta llegó a una decisión luego de una reflexión cuidadosa sobre lo que de hecho sabía y lo que en verdad ignoraba acerca de los hechos que precedieron al juicio."

(D) "Me siento identificada con la frustración de Ana: ahora entiende que el equipo siempre estuvo al tanto de lo inapropiado de la conducta de Juan. Más aún, el propio equipo sabe perfectamente bien que el equipo siempre supo lo que estaba ocurriendo, y no hizo nada para evitarlo." 
Preguntémonos ahora si estaríamos dispuestos a aceptar la verdad de estas afirmaciones si nos enterásemos de que algunos de los miembros del colectivo no acompañaron la actitud grupal, o inclusive si ninguno lo hizo. Lo que muestran List y Pettit es, justamente, que hay escenarios habituales en los cuales no tenemos reparos en aceptar afirmaciones como (A) o (B), aún sin ningún miembro compartiera las creencias grupales en cuestión. Pero no parece haber escenarios igualmente plausibles en los cuales diríamos que (C) o (D) son verdaderas si ningún miembro del grupo hubiera reflexionado conscientemente.

En otras palabras, pareciera que nos resistimos a conceder que existe conocimiento distribuido de que el grupo tiene conocimiento distribuido de que $\mathrm{f}$ a menos que dicho conocimiento de segundo orden esté anclado en agentes individuales. Si queremos capturar esta intuición formalmente, tal vez deberíamos pedir que nuestros modelos validaran los siguientes condicionales:

$$
\begin{aligned}
& M, w \vDash D D \phi \rightarrow E D f, \quad \text { o inclusive } \\
& M, w \vDash D D \phi \rightarrow C D \phi \quad \text { para todo } \phi, w \text { y } M
\end{aligned}
$$

Pero entonces, desde luego, el conocimiento distribuido se trivializa, como ya hemos notado en secciones anteriores. Dicho de otro modo, cuando tratamos con individuos idealmente responsables, el concepto de conocimiento distribuido no trivial se socava a sí mismo.

\section{Un nuevo desideratum. La naturaleza dinámica de los agentes grupales}

El problema que examinamos en la sección anterior nos ayuda a identificar un nuevo desideratum para los agentes ideales: a saber, los fuerza a ajustar sus estados epistémicos individuales de modo de incorporar el conocimiento distribuido del grupo. En otras palabras, el conocimiento distribuido ejerce una presión normativa 'deflacionaria' sobre los individuos idealmente responsables: los obliga a hacerse cargo de lo que conoce el grupo.

La tesis de que existe una presión normativa sobre los agentes individuales puede representarse con la ayuda de un conjunto de reglas dinámicas que capturen el modo en que un modelo inicial evoluciona en el tiempo, generando así una secuencia de modelos. Entonces, en lugar de pedir que los condicionales de la sección anterior sean válidos en un modelo inicial (lo que trivializaría inmediatamente el concepto de conocimiento distribuido), pediremos que exista una sucesión de modelos 
con dominio común $W$, en los cuales las relaciones de accesibilidad se vayan modificando progresivamente.

Para implementar esta idea, consideremos enriquecer el lenguaje con operadores de la lógica de anuncios públicos, con su semántica habitual ${ }^{17}$. Me referiré a dicho lenguaje como $L_{E P}$. Consideremos pues una secuencia $S=\left\langle M_{0}, \ldots M_{k}>\right.$, con dominio común $W$, tal que:

(a) Nuestra secuencia comienza con una estructura $M_{0}$, o modelo base.

(b) Luego, tenemos sucesivos requerimientos de cambios sobre $M_{0}$, motivados por el deseo de alcanzar una agencia individual plenamente responsable, que dé cuenta de las demandas del grupo. Tales cambios pueden originarse a partir de 'anuncios' o comunicaciones hechas de buena fe, esto es, el tipo de fenómeno que ha sido estudiado por las Lógicas de Anuncios Públicos. El efecto típico de tales anuncios es la modificación de las relaciones de accesibilidad de algunos miembros del grupo. Permitir que la información 'circule', digamos, puede provocar cambios en uno o más agentes, generando una secuencia de modelos $M_{1} \ldots M_{k}$. En otras palabras, $M_{1} \ldots M_{k}$ son modelos generados por anuncios públicos hechos de buena fe.

Así, necesitamos encontrar, para cada oración f sobre la cual el grupo tiene conocimiento distribuido en el modelo base (en un mundo $w$ ), una sucesión de oraciones $\psi_{1 \ldots} \psi_{n}$ tales que, luego del anuncio de la oración $\left[\psi_{i}\right], \psi_{i}$ se vuelva verdadera, hasta que llegamos a $C \phi$. Más precisamente:

Para cada oración $\phi$ y mundo $w$ : si $M_{0}, w \vDash D_{\{1 \ldots n\}} \phi$, existen oraciones $\psi_{1} \ldots \psi_{k}$ tales que:

$$
\begin{aligned}
& M_{1}, w \vDash\left[\psi_{1}\right] \phi_{1} \\
& M_{2}, w \vDash\left[\psi_{2}\right] \phi_{2} \\
& \ldots \\
& M_{k}, w \vDash\left[\psi_{k}\right] \phi_{k}, \quad \text { donde } \phi_{k}=C \phi
\end{aligned}
$$

Así pues, para cualquier oración $\phi_{i}$ y mundo $w$, si $M_{i}, w \vDash D_{\{1 \ldots n\}} \phi_{i}$, entonces habrá algún $j \geq i$ tal que $M_{j}, w \vDash C_{\{1 \ldots n\}} \phi_{i}$. En el límite, todo el conocimiento distribuido de $M_{0}$ deviene conocimiento común en algún modelo de la progresión.

Esta tarea dota a las lógicas de anuncios públicos (PA) de una nueva justificación teórica, y a la vez de nuevos objetivos formales. Por un

17 Para una presentación general cf. por ejemplo VAN DITMARSCH, Hans; VAN DER HOEK, Wiebe; KOOI, Barteld, Dynamic Epistemic Logic, Dordrecht: Springer, 2008. 
lado, de ahora en más podemos interpretarlas como herramientas para monitorear el grado de progresión (dentro de un sistema de modelos) hacia la satisfacción plena de los compromisos epistémicos de los individuos involucrados. Por otro lado, y ahora desde un punto de vista técnico, las lógicas PA ahora enfrentan el desafío de encontrar maneras eficientes de construir una ruta desde ' $D \phi$ ' a ' $C \phi$ ', para cada oración $\phi$ y cada mundo, dado que tendremos diferentes maneras de definir la ruta mencionada - o, en otras palabras, diferentes secuencias $S$.

(c) Finalmente, nuevas interacciones de los agentes individuales con el medio puede resultar en cambios ulteriores en sus relaciones de accesibilidad, esta vez para reflejar la incorporación de nuevo conocimiento fáctico (conocimiento sobre proposiciones fácticas), lo cual a su vez generará nuevo conocimiento distribuido. Así, eventualmente obtendremos un nuevo modelo $M_{0}^{\prime} \in S^{\prime}$, momento en el cual todo el proceso recomienza.

Obsérvese que si efectivamente el desideratum 'deflacionario' se satisface, en algún momento reaparecerá la presión inflacionaria que reivindican los argumentos de Pettit y List, una vez que se hayan producido nuevos cambios epistémicos individuales (idea que quedó recogida en el punto (c)). Como consecuencia, obtenemos que los grupos son entidades esencialmente inestables, o, si se prefiere, dinámicas.

Esta inestabilidad se manifiesta como una oscilación entre la generación de creencias y conocimientos más allá de las actitudes intencionales individuales, y la apropiación de las mismas nuevamente por parte de los individuos. Dicho de otro modo, la responsabilidad epistémica ideal del grupo y la de los individuos que lo componen mantienen entre sí una tensión dialéctica: encontramos por un lado una presión inflacionaria, que apunta a permitir creencias grupales que vayan más allá de las individuales, pero una vez que las tenemos aparece inmediatamente otra presión en sentido opuesto. La presión inflacionaria está motivada por consideraciones ontológicas sobre la naturaleza de los grupos, y funciona a la vez como un mecanismo de presión para 'descargar' la responsabilidad individual en el seno del propio grupo. Parece plausible pensar que todos experimentamos en alguna medida esta presión hacia el descargo de responsabilidad en el grupo o grupos de los que formamos parte; de hecho, tendemos a verlos efectivamente como agentes (grupales) en la medida en que ejercen esta presión. La presión opuesta, por su parte, está motivada por consideraciones sobre la responsabilidad epistémica, y por consideraciones ontológicas sobre la naturaleza de las actitudes intencionales de segundo orden. 


\section{El conocimiento grupal revisitado}

Esta manera de concebir la naturaleza de la agencia grupal tiene un corolario inmediato para el conocimiento grupal. En honor a nuestra discusión anterior, sugiero aquí que el conocimiento grupal constituye un fenómeno complejo que no puede ser reducido enteramente a conocimiento común o distribuido - ni tampoco, en realidad, a ninguna otra actitud proposicional, en sentido estricto. Antes bien, sugiero que el conocimiento grupal, en un mundo $w$, es una posible hoja de ruta. El conocimiento grupal en un momento particular no es un conjunto de proposiciones, sino más bien una expectativa de apropiación de un conjunto de proposiciones, o la esperanza de adquirir un grupo particular de proposiciones.

Para decirlo de modo diferente, el conocimiento grupal, en un mundo $w$, es una posible trayectoria: es una tarea a realizar, más que un objeto fijo. Esta es una manera informal y algo metafórica de expresar la idea, pero podemos dar una formulación más precisa. Capturaremos esta idea por medio del siguiente par ordenado:

Para un modelo base $M_{0}$, y un mundo $w$ :

$$
\operatorname{Know}_{G}(w)=<<R D_{0}(w), R C_{0}(w)>, F>
$$

donde ' $R D_{0}(w)$ ' es la proposición más fuerte conocida distribuidamente en $w$ y $M_{0}$; ' $R C_{0}(w)$ ' es la proposición más fuerte de la que se tiene conocimiento común en $w$ y $M_{0}$; y

' $F$ ' refiere a una familia de posibles secuencias $S_{i}=\left\langle M_{0}, \ldots, M_{j}\right\rangle$, definidas del modo indicado anteriormente, que nos llevan de ' $R D_{0}(w)$ ' a ' $R C_{0}(w)$ ', en el siguiente sentido: Para cada $S_{i}$, existe un $M_{j} \in S_{j}$ tal que $R C_{j}(w)$ es la proposición más fuerte de la que se tiene conocimiento común en $w$ en el modelo $M_{j}$; y $R C_{j}(w)=R D_{0}(w)$.

Así, el primer miembro del par ' $\angle\left(R D_{0}(w), R C_{0}(w)>\right.$ ' exhibe la distancia que los agentes ideales deben recorrer para llegar a su meta. Cada trayectoria en $F$ puede verse como una secuencia de pasos (más o menos eficientes) para efectivamente conseguir esta meta, a saber, para conseguir que el conocimiento distribuido coincida con el conocimiento común ${ }^{18}$.

18 Es importante advertir que esta secuencia de pasos no puede servir como un conjunto de instrucciones que puedan ser conscientemente implementadas por los agentes reales: es el téorico el que ve la posibilidad de construir dicha sucesión de modelos. De hecho no hay un único agente que pueda satisfacer por sí mismo los requisitos, ya que su cumplimiento depende del comportamiento agregado de todos los miembros del grupo. Y, desde luego, simplemente decirles a los agentes que se esfuercen por comunicar todo lo que saben resulta 


\section{Conclusiones}

El argumento central de este trabajo puede resumirse de manera breve. Empezamos por aceptar que, para evitar inconsistencias y proceder efectivamente como un agente colectivo, un grupo necesita ser capaz de 'colectivizar la razón', esto es, generar creencias verdaderas que vayan más allá de lo que sabe cada uno de sus miembros; aquí aparece la presión inflacionaria. Esto parece hablar a favor de la idea de interpretar al menos algunas instancias de conocimiento grupal como conocimiento distribuido. Ahora bien, el conocimiento distribuido para agentes idealmente responsables verifica transparencia, lo que garantiza la posibilidad de responsabilidad grupal. Sin embargo, observamos que una actitud grupal de segundo orden sin anclaje individual es anti-intuitiva, en varios aspectos. Esto nos llevó a proponer un nuevo desideratum para los agentes individuales: un agente individual idealmente responsable tenderá a 'anclar' las creencias y conocimiento grupales, modificando apropiadamente su propio corpus epistémico individual; aquí aparece pues lo que llamé una presión deflacionaria. Como consecuencia, podemos caracterizar a los grupos como entidades inestables, o dinámicas. Correlativamente, el conocimiento grupal responsable, en un momento particular, debería interpretarse en términos de una familia de posibles trayectorias entre el conocimiento distribuido y el conocimiento común. Adviértase que de este modo el conocimiento grupal en un momento dado está determinado no solamente por lo que cada agente sabe, sino también por lo que cada uno ignora sobre lo que los otros saben. En este marco, las Lógicas de Anuncios Públicos pueden adoptarse como herramientas que monitorean el grado de progreso hacia la satisfacción de compromisos individuales.

Adviértase, finalmente, que la presión para transformar el conocimiento distribuido en conocimiento compartido (y finalmente en conocimiento común) equivale a una presión para comunicar mutuamente la información particular de que se disponga, e inclusive para deliberar. Normalmente esta presión se conceptualiza como un requisito de conveniencia práctica o prudencial (ya que todos los miembros resultan favorecidos), o inclusive como una exigencia de la moralidad (por ejemplo, para obtener una

impracticable. Una manera de sobreponerse a esta dificultad es pedir a los agentes que revelen sus probabilidades personales acerca de los hechos que el grupo quiera establecer en un momento dado. Si modelamos las probabilidades personales de los agentes en un marco kripkeano, podemos tenerlas también como objeto de los anuncios públicos. Un marco más rico para hablar del conocimiento de un grupo, entonces, incluirá no solamente operadores de conocimiento, sino asignaciones de probabilidad. Por razones de espacio dejaré la reflexión sobre probabilidades grupales para otra oportunidad. 
estructura política bien comportada, equitativa, etc.). La novedad de la presente perspectiva radica en que, según espero haber mostrado, dicha presión aparece primariamente como una restricción epistemológica y metafísica motivada por la inevitabilidad de la emergencia de actitudes grupales de segundo orden. Un agente epistémicamente responsable está sujeto a dicha presión por mera coherencia conceptual.

En este punto podrían plantearse dos posibles objeciones. Una de ellas concierne a un problema práctico. A saber, podría objetarse que no es una buena idea gastar recursos intentando que los individuos se apropien (simbólicamente) de lo que el grupo sabe en sentido distribuido; después de todo, es justamente la posibilidad del conocimiento distribuido lo que hace que nuestras comunidades sean más eficientes. Frente a esta observación cabe la siguiente reflexión. Nadie negaría que invertir algún tiempo en interiorizarse del saber de otros es ciertamente saludable (y típicamente se lo incentiva, aunque no más sea por la expectativa normal de envejecimiento - y su consecuente necesidad de reemplazo - de los miembros de un grupo). Sin embargo, dado que los recursos de todo grupo son limitados, es claro que algunas líneas de colaboración y transmisión de información deberán tener prioridad sobre otras. El análisis ofrecido en este trabajo no contradice esta afirmación, sino que plantea un ideal de conocimiento grupal responsable.

De manera aún más drástica, podría cuestionarse el ideal mismo. Podría alegarse que hay otros ideales que interfieren con la perspectiva de libre intercambio de conocimiento. Por ejemplo, habrá grupos en los que existe el deber de no revelar información sensible a otros miembros. Pero los casos de conflictos de deberes ocurren en todo tipo de circunstancias, y aquejan de manera general la aplicación de cualquier tipo de teorías (ya sea morales, políticas, o epistemológicas) a los agentes reales; no se trata pues de un problema especial del conocimiento grupal.

En efecto, cuando un agente real se ve enfrentado a la necesidad de cumplir a la vez con deberes morales, deberes metafísicos, y deberes epistemológicos, entre otros posibles, bien puede ocurrir que exista tensión entre ellos. Podría intentarse aquí alguna reflexión general acerca de la manera en que estas esferas se relacionan entre sí; por ejemplo, podríamos preguntarnos si alguna esfera tiene prioridad sobre las otras, ya sea en términos absolutos o dependiendo del contexto. Esta es una idea interesante para explorar en el futuro. Por el momento, la presente propuesta tiene la virtud de exponer de manera clara hasta qué punto los deberes involucrados en la transmisión de información pertenecen a ámbitos que normalmente no se han asociado con este problema. Se ha dado así un primer paso para pensar con mayor seriedad la relación entre consideraciones prudenciales, morales, políticas, metafísicas y 
E. Cresto - El conocimiento grupal de agentes ...

epistemológicas, todas las cuales se hallan involucradas, de una u otra manera, en la reflexión sobre la agencia y el conocimiento grupal.

\section{Referencias}

CRESTO, Eleonora. A Defense of Temperate Epistemic Transparency. In: Journal of Philosophical Logic, 41:6 (2012), p. 923-955.

DE BRUIN, Boudewijn. Epistemic Logic and Epistemology. In: HENDRICKS, Vincent; PRITCHARD, Duncan (eds.). New Waves in Epistemology. New York: Palgrave Macmillan, 2008. p. 106-136.

FAGIN, Ronald; HALPERN, Joseph Y.; MOSES, Yoram; VARDI, Moshe Y. Reasoning About Knowledge. Cambridge, Mass.: MIT Press, 1995.

GILBERT, Margaret. On Social Fact.s. London-New York: Routledge, 1989.

KORNHAUSER, L. A.; SAGER, L. G. Unpacking the Court. In: Yale Law Journal, 96 (1986), p. 82-117.

LACKEY, Jennifer. Group Belief: Lessons from Lies and Bullshit. In: IDEM. The Epistemology of Groups. Oxford: Oxford University Press, en prensa.

LIST, Christian; PETTIT, Phillip. Group Agency: The Possibility, Design, and Status of Corporate Agents. Oxford: Oxford University Press, 2011.

PETTIT, Philip. Groups with Minds of Their Own. In: SCHMITT, Frederick (ed.). Socializing Metaphysics. New York: Rowman and Littlefield, 2003. p. 167-193.

TUOMELA, Raimo. “Group Beliefs”. In: Synthese, 91 (1992), p. 285-318.

VAN DITMARSCH, Hans; VAN DER HOEK, Wiebe; KOOI, Barteld. Dynamic Epistemic Logic. Dordrecht: Springer, 2008. 